\title{
Deteção da radiação térmica emitida por um filamento de tungstênio aquecido
}

Detection of the thermal radiation emitted by a hot tungsten filament

\author{
Luis Peralta*1,2, Florbela Rego ${ }^{2}$ \\ ${ }^{1}$ Faculdade de Ciências, Universidade de Lisboa, Lisboa, Portugal \\ ${ }^{2}$ Laboratório de Instrumentação e Física Experimental de Partículas, Universidade de Lisboa, Lisboa, Portugal
}

Recebido em 21 de julho de 2015. Aceito em 17 de outubro de 2015

\begin{abstract}
A demonstração da lei de Stefan-Boltzmann recorrendo a uma lâmpada de incandescência como corpo negro já se tornou uma experiência clássica. Neste artigo exploramos a utilização de fotodíodos de baixo custo como fotodetetores neste tipo de experiência e apresentamos uma discussão dos resultados obtidos com base na distribuição espetral de Planck.

Palavras-chave: lei de Stefan-Boltzmann, distribuição de Planck, fotodíodos.
\end{abstract}

The demonstration of the Stefan-Boltzmann law using a hot tungsten filament has become a classical experiment. In this article we investigate the use of low cost photodiodes as the photodetectors of the experiment. The obtained results are discussed in the framework of Planck's spectral distribution.

Keywords: Stefan-Boltzmann law, Planck's distribution, photodiodes.

\section{Introdução}

Um corpo que se encontre a uma temperatura diferente de zero kelvin irá radiar energia sob a forma de radiação eletromagnética. Para um corpo de propriedades ideais, conhecido como corpo negro, a distribuição espectral da potência emitida por unidade de área é descrita pela distribuição de Planck $[1,2]$

$$
\frac{d R}{d \lambda}=\frac{2 \pi h c^{2}}{\lambda^{5}\left(e^{h c / \lambda k T}-1\right)}
$$

onde $h$ é a constante de Planck, $c$ a velocidade da luz, $k$ a constante de Boltzmann, $\lambda$ o comprimento de onda e $T$ a temperatura absoluta do corpo. A potência total radiada pelo corpo negro por unidade de área é assim obtida por integração da distribuição espectral para todos os comprimentos de onda $[1,2]$

$$
R=\int \frac{d R}{d \lambda} d \lambda=\sigma T^{4}
$$

obtendo-se a conhecida lei de Stefan-Boltzmann onde $\sigma=5,6704 \times 10^{-8} \mathrm{Wm}^{-2} \mathrm{~K}^{-4}$. A potência

*Endereço de correspondência: luis@lip.pt. por unidade de área radiada por um corpo real à temperatura $T$, é menor que a prevista pela lei de Stefan-Boltzmann para o corpo negro, pelo que se introduz a grandeza emissividade $\varepsilon$, tal que

$$
R=\varepsilon \sigma T^{4} .
$$

Neste trabalho iremos descrever algumas experiências sobre radiação térmica que podem ser realizadas por estudantes do Ensino Médio ou superior utilizando dispositivos de baixo custo e que podem inclusive ser construídos pelos próprios estudantes.

\section{O fio de tungsténio como radiador}

Desde há várias décadas que foi reconhecido que o filamento de tungsténio de uma lâmpada de incandescência poderia ser utilizado como radiador sendo usado em experiências de verificação da lei de StefanBoltzmann [3-8]. Uma das principais vantagens da sua utilização é a possibilidade de controlo e medida da temperatura do filamento a partir das medidas 
da diferença de potencial aplicada e da intensidade de corrente que o percorre.

Para pequenas variações de temperatura a resistividade $\rho$ de um fio metálico pode ser aproximada pela expressão

$$
\rho=\rho_{0}\left(1+\alpha\left(T-T_{0}\right)\right)
$$

em que $\alpha$ é o coeficiente térmico de resistência, e $\rho$ a resistividade à temperatura $T$. Para o tungsténio à temperatura ambiente $\alpha=0,0053 \mathrm{~K}^{-1}[4,8]$.

Para variações da ordem das dezenas ou centenas de kelvin, esta lei não é válida sendo necessário recorrer a tabelas de valores medidos ou a expressões empíricas ajustadas a esses dados [9]. Várias parametrizações da dependência da resistividade na temperatura têm sido propostas com base nesses valores. Uma forma particularmente útil para a obtenção da temperatura a partir da medida da resistência elétrica do filamento de tungsténio é

$$
T=T_{0}\left(\frac{R}{R_{0}}\right)^{\beta} .
$$

No nosso trabalho recorremos à tabela publicada por Desai et al. [10]. Fazendo o ajuste a esses dados usando a Eq. (5) obtemos os valores $T=297,7 \mathrm{~K} \mathrm{e}$ $\beta=0,8087$ sendo o valor de referência da resistência $R$ medido à temperatura de $300 \mathrm{~K}$.

A medida da temperatura do filamento pode ser negativamente afetada por um valor incorreto da resistência à temperatura ambiente. Em particular, no caso em que esse valor é da ordem de apenas alguns ohm, a incerteza cometida quando a medição é realizada com um ohmímetro de baixo custo pode ser bastante elevada ( $10 \%$ ou maior) comprometendo os valores obtidos de temperatura. Assim no caso de se utilizar um ohmímetro digital comum (resolução típica igual a $0,1 \Omega$ ) na experiência devem ser utilizadas de preferência lâmpadas de resistência ohmíca o mais elevada possível (superior a $10 \Omega$ ) de forma obtermos incertezas da ordem do 1 a $2 \%$. No caso se empregarem lâmpadas de baixo valor de resistência (por exemplo de 1 a $5 \Omega$ ) deverá ser utilizado um ohmímetro com boa resolução (0,01 $\Omega$ ou melhor).

Os fabricantes não fornecem normalmente o valor da resistência elétrica das lâmpadas, mas a tensão de trabalho e a potência dissipada ou a intensidade de corrente. Usando a Eq. (5) e a relação $R=V^{2} / P$ podemos obter a resistência $R$ da lâmpada à temperatura ambiente $T$ a partir do valor da tensão $V$ e da potência dissipada $P$

$$
R_{0}=\left(\frac{V^{2}}{P}\right)\left(\frac{T}{T_{0}}\right)^{-1 / \beta} .
$$

Desta relação verificamos que para um determinado valor de tensão de trabalho da lâmpada, quanto menor for a potência dissipada maior é a sua resistência à temperatura ambiente. A temperatura de trabalho das lâmpadas varia num intervalo relativamente largo (2000 a $3000 \mathrm{~K}$ ) pelo que não é possível à priori estabelecer só com os valores de $V$ e $P$ qual o valor de $R$. Assim a Eq. (6) serve apenas para dar uma indicação da lâmpada a escolher.

Devido ao baixo valor da resistência de uma lâmpada, as resistência parasitas podem influenciar a medição do seu valor. Assim, a medição da resistência da lâmpada não deve ser efetuada utilizando as pontas de prova do multímetro, mas soldando directamente fios de calibre adequado à lâmpada, na ponta dos quais se colocam fichas banana, para a ligação ao aparelho de medida. Em virtude dos baixos valores envolvidos, a resistência elétrica dos fios de ligação também devem ser medida separadamente da lâmpada de forma a que o seu valor possa ser descontado.

\section{Construção do sistema de deteção}

Os fotodíodos de silício (do tipo PIN [11]) são sensíveis a uma gama de comprimentos de onda que vai do ultravioleta próximo $(\approx 300 \mathrm{~nm})$ até ao infravermelho próximo $(\approx 1200 \mathrm{~nm})$. Existem no mercado fotodíodos de baixo custo que permitem a realização de uma forma fácil de montagens para demonstrações experimentais. Neste trabalho usámos os fotodíodos BP104 (infravermelho) e BPW34 (visível e infravermelho). Para a amplificação do sinal optámos por construir um amplificador de transimpedância utilizando o integrado LM324. Este integrado é polarizado apenas com uma fonte de alimentação (que pode ser por exemplo uma pilha de $9 \mathrm{~V}$ ) o que é uma vantagem para a sua utilização na escola. $\mathrm{O}$ sinal em corrente produzido no fotodíodo é amplificado e convertido numa tensão, que é lida à saída do amplificador com um voltímetro digital. O esquema de principio do amplificador é apresentado na Fig. 1. A resistência $R_{f}$ de realimentação controla o ganho do amplificador, dado que a tensão de saída é dada pela relação $V_{\text {out }}=I_{f} R_{f}$ [11], sendo $I_{f}$ a corrente à entrada do amplificador. Para o tipo de aplicação deste trabalho um valor da ordem de 


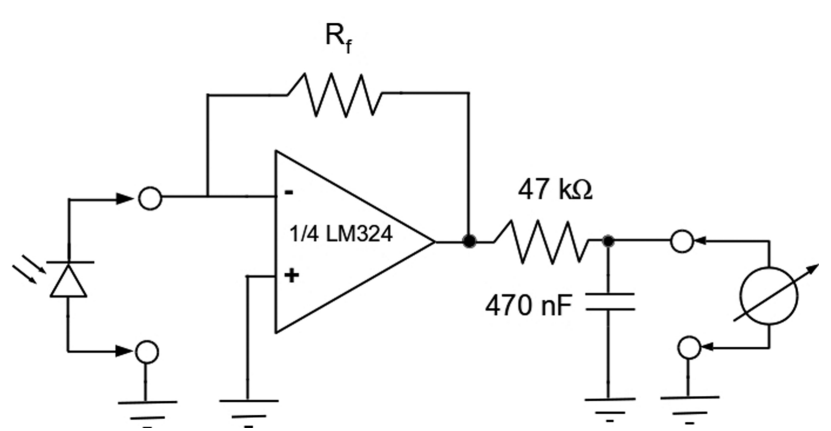

Figura 1: Esquema do amplificador de transimpedância usado para ler o sinal do fotodíodo.

$100 \mathrm{k} \Omega$, mostrou ser adequado para $R_{f}$, pois obtivemos na tensão de saída valores que podiam chegar a vários volt. O filtro RC passa-baixo pretende eliminar interferências que possam perturbar o valor lido à saída, não sendo críticos os valores dos componentes. Este tipo de amplificador foi utilizado para a leitura dos fotodíodos BP104 e BPW34. A sua montagem pode ser realizada de forma fácil sobre uma placa de ensaio (breadboard em inglês [12]) ou sobre uma placa de circuito impresso pré-perfurada [13] no caso de pretendermos uma montagem com maior durabilidade.

\section{Montagem experimental}

Para a realização da experiência será conveniente evitar a interferência da luz ambiente. Escolhemos então um tubo de plástico, com $20 \mathrm{~cm}$ de comprimento, dos vulgarmente usados em instalações elétricas. Para garantir a total ausência de luz ambiente no seu interior, envolvemos o tubo em folha de alumínio. A lâmpada de incandescência de baixa

$$
\text { Voltímetro }
$$

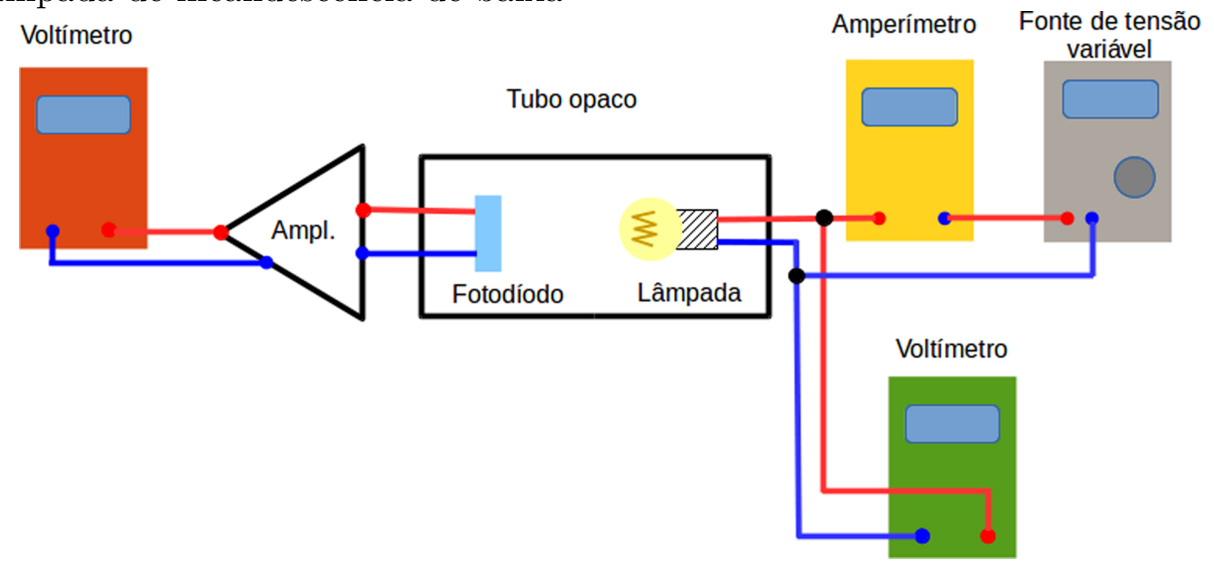

tensão (do tipo usado nas lanternas de bolso) foi colocada numa extremidade do tubo e o fotodíodo na outra extremidade. Rolhas, feitas em espuma de alta densidade foram colocadas nas extremidades do tubo para impedir a saída da lâmpada e fotodíodo.

A lâmpada foi ligada em série com um amperímetro digital para a medida da intensidade de corrente. Este conjunto foi ligado a uma fonte variável de baixa tensão. Em paralelo com a lâmpada foi colocado um voltímetro digital para a leitura da queda de tensão. O fotodíodo foi ligado ao amplificador anteriormente descrito, sendo a saída deste ligada a um voltímetro digital. O esquema da montagem experimental usada é apresentado na Fig. 2. Na Fig. 3 apresentamos uma fotografia do dispositivo com todos os elementos necessários à realização da experiência.

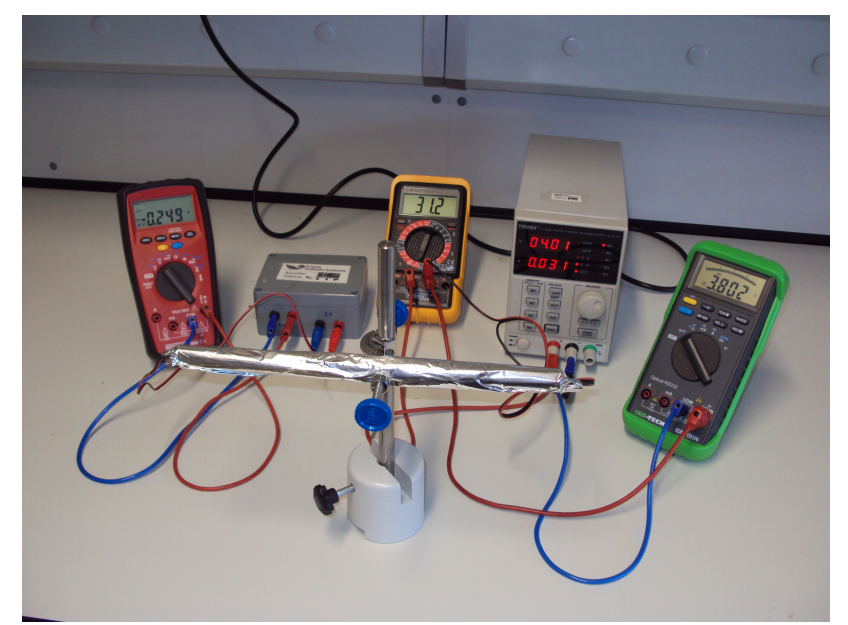

Figura 3: Fotografia da montagem experimental. O fotodíodo e a lâmpada foram colocados dentro de um tubo de plástico envolvido em folha de alumínio para o tornar completamente opaco à luz ambiente. 


\section{Análise dos dados}

Se utilizarmos um detetor em que a janela de sensibilidade cobre apenas uma porção restrita do espetro de comprimentos de onda, apenas detetamos uma fração da potência radiada pela fonte. Assim em geral não iremos obter uma dependência da potência radiada com a quarta potência da temperatura. Nesse caso a potência radiada por unidade de área será dada pelo integral da distribuição espectral de potência por unidade de área na janela de comprimentos de onda correspondente à janela de sensibilidade do fotodetector

$$
\begin{array}{r}
R=\varepsilon \int_{\lambda_{1}}^{\lambda_{2}} S(\lambda) \frac{d R}{d \lambda} d \lambda= \\
\varepsilon \int_{\lambda_{1}}^{\lambda_{2}} S(\lambda) \frac{2 \pi h c^{2}}{\lambda^{5}\left(e^{h c / \lambda k T}-1\right)} d \lambda,
\end{array}
$$

em que $S(\lambda)$ é a sensibilidade do fotodetector.

Os fotodetetores usados foram os fotodíodos BP104 e o BPW34 que são de baixo custo (o preço típico varia entre 1 a 2 euros). O primeiro fotodíodo cobre uma janela de comprimentos de onda no infravermelho (800-1100 nm) e o segundo cobre uma gama de comprimentos de onda que se estende do visível ao infravermelho (430-1100 nm).

Na Fig. 4 está representada a distribuição de Planck para três valores de temperatura $(1500,2250$ e $3000 \mathrm{~K}$ ) e sobrepostas a tracejado as janelas de sensibilidade dos fotodíodos BP104 e BPW34.

Os fotodíodos não possuem uma sensibilidade constante em toda a janela de comprimentos de

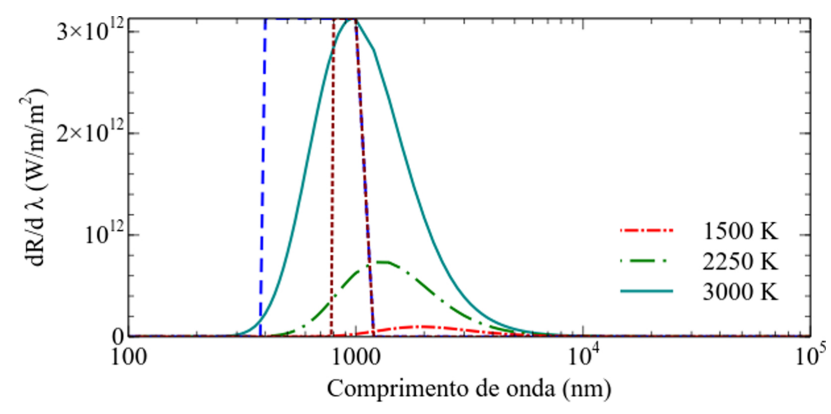

Figura 4: Distribuição de Planck para 3 valores de temperatura: 1500,2250 e $3000 \mathrm{~K}$. A tracejado encontra-se a janela de sensibilidade de cada um dos fotodíodos: BP104 linhas vermelhas tracejado pequeno e BPW34 linhas azuis tracejado largo. onda tendo então as curvas de sensibilidade sido parametrizadas de forma aproximada por polinómios de grau 3 a partir da curva fornecida pelo fabricante dos fotodíodos. Na Tabela 1 encontram-se o valor dos coeficientes dos polinómios utilizados. De notar que esta parametrização apenas reproduz de uma geral o comportamento da sensibilidade dos fotodíodos, não pretendendo ser um ajuste fiel das respetivas curvas, para o qual teríamos que utilizar polinómios de ordem superior. O procedimento para a obtenção da parametrização a partir das curvas dos fabricantes foi o seguinte: começámos por digitalizar o gráfico da sensibilidade do fotodíodo. A figura assim obtida foi transferida para uma folha de cálculo. Foi então sobreposto um gráfico $x y$ de idênticas dimensões e de escalas em $x$ e $y$ idênticas ao gráfico do fabricante. Para um conjunto de 30 valores do comprimento de onda (eixo do $x$ ) foram escolhidos valores de sensibilidade (eixo do $y$ ) de forma a que os correspondentes pontos no gráfico coincidam com a curva do fabricante. Uma vez obtido este conjunto de ponto é efetuado aos mesmos um ajuste polinomial.

Para janelas de comprimento de onda pequenos (como as dos fotodíodos usados) o integral da Eq. (7) pode ser aproximado por um somatório (integração por trapézios)

$$
R=\varepsilon \sum_{k=1}^{n} S\left(\lambda_{k}\right) \frac{2 \pi h c^{2}}{\lambda_{k}^{5}\left(e^{h c / \lambda_{k} k T}-1\right)} \Delta \lambda,
$$

em que o intervalo de comprimentos de onda $\lambda_{n}-\lambda_{1}$ é dividido em $\mathrm{n}$ subintervalos de largura $\Delta \lambda=\left(\lambda_{n}-\lambda_{1}\right) / n$ sendo as funções calculadas nos $\mathrm{n}$ pontos intermédios $\lambda_{k}=\lambda_{1}+$ $(k-1 / 2) \Delta \lambda \quad(k=1, n)$ de cada um dos subintervalos . Este tipo de somatório pode ser facilmente

Tabela 1: Coeficientes do polinómio $S(\lambda)=A_{0}+A_{1} \lambda+$ $A_{2} \lambda^{2}+A_{3} \lambda^{3}$ de ajuste das curvas de sensibilidade dos fotodíodos BP104 e BPW34 (comprimento de onda $\lambda$ em metros).

\begin{tabular}{lll}
\hline & BP104 & BPW34 \\
\hline A0 & $-1,0528 \times 10^{2}$ & $+3,3614 \times 10^{-1}$ \\
A1 & $+2,9482 \times 10^{8}$ & $-4,7602 \times 10^{6}$ \\
A2 & $-2,6710 \times 10^{14}$ & $+1,3272 \times 10^{13}$ \\
A3 & $+7,8413 \times 10^{19}$ & $-8,1207 \times 10^{18}$ \\
\hline
\end{tabular}


obtido numa folha cálculo sendo a incerteza obtida com um passo $\Delta \lambda=10 \mathrm{~nm}$ menor que $0,1 \%$.

\section{Resultados}

Na Fig. 5 estão os resultados obtidos para os fotodíodos BP104 e BPW34 com uma lâmpada de resistência $R=15,1 \Omega$. O somatório foi obtido com o recurso a uma folha de cálculo e utilizou-se um passo $\Delta \lambda=10 \mathrm{~nm}$. Para a emissividade do filamento foi considerado um valor constante. Para efeitos de comparação entre os dados e a curva teórica, normalizamos ambas as curvas ao ponto de temperatura aproximadamente igual a $2000 \mathrm{~K}$. São apresentadas as curvas considerando as sensibilidades como constantes na janela de comprimentos de onda dos fotodíodos ou variando de acordo com as funções dadas na Tabela 1. Tal como pode ser verificado a partir dos gráficos o acordo obtido entre a previsão teórica e os dados obtidos experimentalmente é muito boa.

Para temperaturas elevadas a janela de sensibilidade do fotodíodo BPW34 cobre uma porção importante do espectro de emissão térmico (ver Fig. 4). Para este intervalo de temperaturas o sinal dado pelo fotodíodo tem um comportamento que se aproxima de uma variação com a quarta potência da temperatura (Fig. 6). Contudo, não é recomendável elevar a temperatura muito acima dos 3000 K. Em-
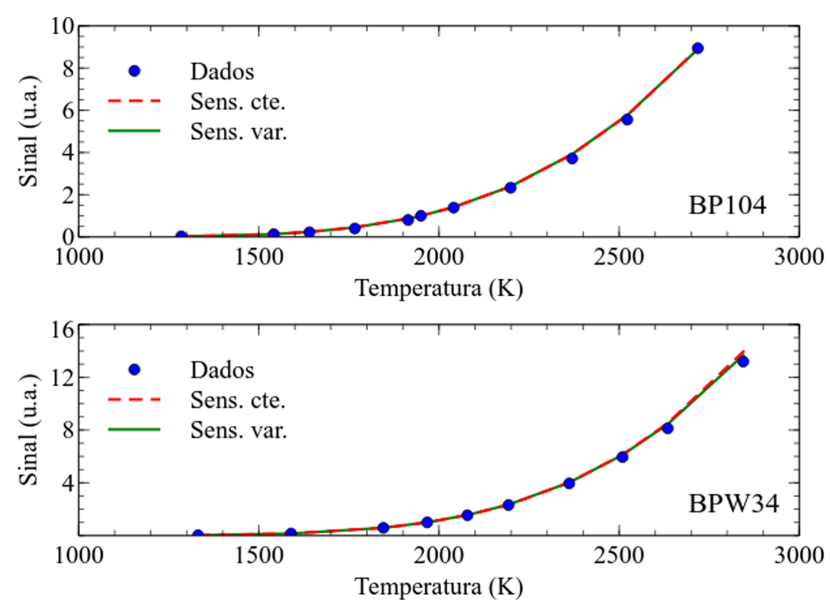

Figura 5: Sinal obtido com os fotodíodos BP104 e BPW34 em função da temperatura. As curvas são a previsão teórica da distribuição de Planck com sensibilidade constante (curva vermelha na versão eletrônica) e sensibilidade dada por uma das curvas da Tabela 1 (curva verde na versão eletrônica). As curvas foram normalizadas ao valor do sinal correspondente à temperatura de aproximadamente $2000 \mathrm{~K}$.

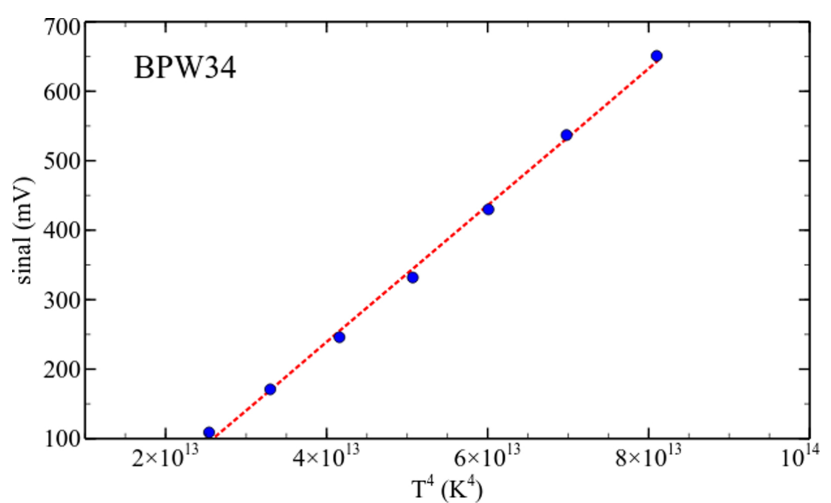

Figura 6: Sinal obtido com o fotodíodo BPW34 em função da quarta potência da temperatura $T^{4}$, no intervalo entre 2200 e 3000 K. A linha a tracejado é um ajuste linear aos dados.

bora o ponto de fusão do tungsténio se encontre a $3695 \mathrm{~K}$, existe o perigo de antes de se atingir essa temperatura o filamento começar a vaporizar, indo depositar-se no vidro da lâmpada. Esta irá adquirir um aspecto acinzentado, perdendo parte da sua transparência, em consequência deixando passar uma menor quantidade de radiação, comprometendo os resultados obtidos.

\section{Conclusão}

Esta experiência permite mostrar aos alunos diversos aspectos de uma medida experimental. Desde logo o facto do instrumento de medida ter um alcance limitado (em comprimento de onda) o que influencia de forma decisiva a medida que é possível obter. A sensibilidade em função do comprimento de onda do fotodíodo não é uniforme o que introduz uma pequena distorção na curva medida face ao previsto. Essa distorção pôde ser corrigida introduzindo uma função de sensibilidade do fotodíodo que, embora aproximada, permite obter uma curva mais próxima dos dados. O tratamento dos dados conduz também ao conceito de cálculo aproximado de um integral por métodos numéricos elementares.

Uma última palavra sobre sistema de deteção da luz. Existem no mercado conversores integrados luztensão como seja o caso dos TSL12S/13S/14S [14] que podem ser utilizados nesta experiência. Estes integrados necessitam apenas de uma fonte de alimentação (por exemplo uma pilha de $4,5 \mathrm{~V}$ ) e a sua utilização é muito fácil uma vez que não necessitam de componentes exteriores para o seu funcionamento. Em particular, a janela de comprimento de onda 
acessível com estes fotodetetores é semelhante à do fotodíodo BPW34. Em virtude também do seu baixo custo (cerca de 1,5 euros por unidade) são uma excelente opção para a realização deste trabalho.

\section{Agradecimentos}

São devidos agradecimentos à Agência Nacional para a Cultura Científica e Tecnológica de Portugal pelo apoio financeiro dado. Agradecemos igualmente a todos os colegas participantes no Projeto Radiação Ambiente que realizaram a experiência com seus alunos do Ensino Secundário e cujas sugestões permitiram melhorar esta atividade. Finalmente agradecemos ao Prof. Nilson Garcia pelos incentivos à escrita e comentários ao artigo.

\section{Referências}

[1] J.W. Rohlf, Modern Physics from a to Z0 (John Wiley and Sons, New York, 1994).

[2] R.A. Serway and J.W. Jewett, Physics for Scientists and Engineers with Modern Physics (Cengage Learning, Boston, 2013), 9th ed.

[3] I.R. Edmonds, American Journal of Physics 36, 845 (1968).

[4] B.S.N. Prasad and Rita Mascarenhas, American Journal of Physics 46, 420 (1978).

[5] 36th International Olympaid, Salamanca, webpage http://www.jyu.fi/tdk/kastdk/olympiads/ 2005/Exp.pdf acesso em 2015.

[6] Imtiaz Ahmad, Sidra Khalid and Ehsan E. Khawaja, Lat. Am. J. Phys. Educ. 474 (2010).

[7] Marcello Carla, Am. J. Phys. 81, 512 (2013).

[8] Francisco J. Abellan, Jose A. Ibañez, Ramon P. Valerdi and Jose A. Garcia, Eur. J. Phys. 34, 1221 (2013).

[9] V. Zanetti, Am. J. Phys. 53, 546 (1985).

[10] P.D. Desai, T.K. Chu, H.M. James and C.Y. Ho, J. Phys. Chem. Ref. Data 13, 1069 (1984).

[11] Thomas L. Floyd, Electronic Devices, Conventional Current Version (Prentice Hall, Boston, 2012), 9th ed.

[12] http://pt.wikipedia.org/wiki/Placa_de_ Ensaio, acesso em 2015.

[13] http://en.wikipedia.org/wiki/Stripboard acesso em 2015.

[14] http://www.ece.usu.edu/ece_store/spec/ 856-TSL14S-LF.pdf, acesso em 2015. 